\title{
Fecal Microbiota Transplantation (FMT)
}

\section{What is the Future of Fecal Transplantation? Questions to be}

\section{Asked}

\author{
Aziz Koleilat* \\ Department of Pediatrics, MUGH, Beirut Arab University, Lebanon \\ *Corresponding author: Aziz Koleilat, Department of Pediatrics, Makassed University \\ General Hospital, Beirut Arab University, Beirut, Lebanon, Tel: 009613231717; E-mail: \\ drkoleilat@gmail.com
}

Keywords: Microbiota; Fecal transplant; C. Defficili; Probiotics

Abbreviations: FMT: Fecal microbiota transplantation; CD: Clostridium difficile; FT: Fecal transplant; NCD: Non communicable diseases.

\section{Introduction}

Fecal microbiota transplantation (FMT) is the transfer of stool from a "healthy" donor to a recipient believed to harbor an altered colonic microbiome resulting in disease [1].

The goal is to restore eubiosis, or a "healthy" microbiome which was distorted by diet, antibiotics, inflammations and other causes.

Often referred to as, Stool trans- plantation, Fecal transplantation, Fecal flora reconstitution, Fecal bacterio therapy, Human probiotic infusion [1-3].

The rationale of using FMT to treat disease has been validated by its successful use in treating recurrent $C$. difficile infection.

C. difficile occurs as a result of decreased microbial diversity in the gut, and impaired microbial community resilience and colonization resistance, most often in the setting of recent antibiotic treatment.
Large majority of patients with C. difficile achieved complete cure of infection by FMT [4-6].

Fecal transplant was first documented in the 4th century in China, which was known as "yellow soup", It was made of fecal matter and water, and was given to the patient with intestinal infections.

The Chinese medical literature mentions its use for treating food poisoning and severe diarrhea.

Li Shizhen, (The 16th century Chinese physician) used "yellow soup,"'"golden syrup," containing fresh, dried, or fermented stool to treat abdominal diseases [2,7].

Used by German soldiers in Africa to treat dysentery during World War II. 1940. First time was described in the west, in 1958 by Ben and colleagues, who successfully treated four critically ill patients with fulminant pseudomembranous colitis using fecal enemas [8].

In 1959, another successful fecal transplant for UC (ulcerative colitis) was done by Bennet and colleagues (it was given to himself) [9].

The therapeutic potentials of FMT in nongastroenterologic conditions being studied [10-13].

These conditions include: Auto immune disorders, obesity, increased insulin sensitivity, food allergy, metabolic syndrome, diabetes, neurological conditions, 


\section{Gastroenterology \& Hepatology International Journal}

multiplesclerosis, parkinson's disease, tumors, With promising results mainly in adults.

The future holds a lot of promise for the potential applications for FMT in obesity, NAFLD, CDI, IBD and Autisim.

A key question now arises: Could we manipulate the microbiota environment to treat or prevent obesity in humans especially children?

Can FMT play a role in pediatric IBS, inflammatory bowel disease, atopy and inflammation, autismwhich has been shown also to have a disturbed microbiome which is fairly common and difficult to treat? [14].

Does future advancement in delivery of FMT will soon allow commercial use of a capsule form of FMT with desiccated microorganisms?

Do we need all the microorganisms or can we tailor them to a specific disease? [15].

Would Bacteroides (human probiotics) alone be sufficient to cure patients since they seem to be key organisms these diseases? [16].

Can we optimize microbiota to prevent infections or to reconstitute the microbiota following antibiotic treatment?

If this is possible, what is the time frame for this to be needed...till now it is not clear.

Must the administration of microbiota components be matched to the host's genotype, diet, or environment!!?

Since some specific commensal bacterial species might cause disparate immune responses in different individuals.

To what extent can dietary changes optimize the intestinal microbiota, and how will this influence the immune system? [17].

Although our understanding of the microbiome and mucosal immune system is moving forward rapidly and could be explained.

The diversity of the fecal microbiota and the marked genomic variation even within well-defined bacterial species is making the design of optimal probiotic combinations challenging!!!
Eventually, with the development of probiotic combinations, fecal transplantation will be replaced by administration of probiotic consortia.

Diet has been demonstrated to alter the microbiota and enhance the ability of the microbiota to absorb calories, and this is likely to also extend to relative immune activation.

Knowing the impact of the microbiota on gutassociated immune tissues and systemic immune development, this will remain the focus research for many years to come.

Summing up all the above we can say:

Fecal microbiota transplant (FMT) has been introduced several decades ago in an attempt to restore the gut microbial balance.

FMT appears to be the most efficient method to effectively change and sustain the gut microbial composition.

Success in eliminating recurrent Clostridium difficile infections and restoring the gut microbial profile to resemble that of the healthy donor in this difficult to treat population is $90 \%$.

The new gut microbiome appears to be stable in the recipients for at least 24 weeks [2].

FMT is still difficult for the pediatric scientific community to embrace andaccept this therapy as there are only sporadic reports in children.

FMT has also been used now to treat inflammatory bowel disease, especially ulcerative colitis and was successful [18].

FMT may be a treatment of many difficult diseases.

FMT could be adjuvant for the treatment of noncommunicable diseases.

FMT will be the future of treatment, who knows, it may replace antibiotics!!!!! [2]

\section{References}

1. Stephen M Vindigni, Christina M Surawicz (2017) Fecal Microbiota Transplantation. Gastroenterology Clinics 46(1): 171-185. 


\section{Gastroenterology \& Hepatology International Journal}

2. Michail S, Klonoff A (2015) Review of Fecal Transplant in Childhood Gastrointestinal Disorders. Ann Pediatr Child Health 3(2): 1043.

3. Bakken JS, Borody T, Brandt LJ, Joel V Brill, Daniel C Demarco, et al. (2011) Treating Clostridium difficile infection with fecal microbiota transplantation. Clin Gastroenterol Hepatic 9(12): 1044-1049.

4. Thomas Malikowski, Sahil Khanna, Darrell S Pard (2017) Fecal Microbiota Transplantation for Gastrointestinal Disorders. Curr Opin Gastroenterol 33(1): 8-13.

5. Khanna S, Pardi DS (2012) Clostridium difficile infection: new insights into management. Mayo Clin Proc 87(11): 1106-1117.

6. Choi HH, Cho YS (2016) Fecal Microbiota Transplantation: Current Applications, effectiveness, and future perspectives. Clin Endosc 49(3): 257-265.

7. Li Shizhen $16^{\text {th }}$ century Chinese physician, herbalist, and acupuncturist.

8. Eiseman B, Silen W, Bascom GS, Kauvar AJ (1958) Fecal enema as an adjunct in the treatment of pseudomembranous enterocolitis. Surgery 44(5): 854-859.

9. Bennet JD, Brinkman M (1989) Treatment of ulcerative colitis by implantation of normal colonic flora. Lancet 333(8630): 164.

10. Borody TJ, Khoruts A (2012) Fecal microbiota transplantation and emerging applications. Nature reviews gastroenterology and Hepatology 9(2): 8896.
11. Borody T, Leis S, Campbell J, Torres M, Nowak A (2011) Fecal microbiota transplantation (FMT) in multiple sclerosis (MS). American Journal of Gastroenterology 106: S352-S352.

12. Olga C Aroniadis Lawrence J Brandt (2013) Fecal Microbiota Transplantation. Curr Opin Gastroenterol 29(1): 79-84.

13. Meng-Que Xu, Cao HL, Wang WQ, Wang S, Cao XC, et al. (2015) Fecal microbiota transplantation broadening its application beyond intestinal disorders. World J Gastroenterology 21(1): 102-111.

14. Rigsbee L, Agans R, Shankar V, Kenche H, Khamis HJ, et al. (2012) Quantitative profiling of gut microbiota of children with diarrhea-predominant irritable bowel syndrome. Am J Gastroenterol 107(11): 17401751.

15. Tvede M, Rask Madsen J (1989) Bacteriotherapy for chronic relapsing Clostridium difficile diarrhoea in six patients. Lancet 1(8648): 1156-1160.

16. Schubert AM, Rogers MA, Ring C, Mogle J, Petrosino JP, et al. (2014) Microbiome data distinguish patients with Clostridium difficile infection and non-C. difficile-associated diarrhea from healthy controls. $\mathrm{M}$ Bio 5(3): e01021-01014.

17. Pamer, Michael C Abt (2014) Commensal bacteria mediated defenses against pathogens. Curr Opi Immunol 29: 16-22.

18. Carl Crawford, Randy Longman, Vinita Jacob (2017) Fecal Microbiota Transplant is Safe and Effective for Patients with Ulcerative Colitis. Weill Cornell Medicine.

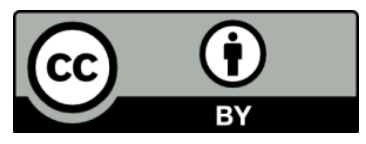

